\section{Intersections}

Canadian Journal of Music

Revue canadienne de musique
Intersections CANADIAN JOURAL OF MUSIC
REVUE CANADIENEE DE MUSIOUH

\title{
Relire Ingarden : l'ontologie des oeuvres musicales, entre fictions et montagnes
}

\section{Esteban Buch}

Volume 32, numéro 1-2, 2012

URI : https://id.erudit.org/iderudit/1018579ar

DOI : https://doi.org/10.7202/1018579ar

Aller au sommaire du numéro

\section{Éditeur(s)}

Canadian University Music Society / Société de musique des universités canadiennes

\section{ISSN}

1911-0146 (imprimé)

1918-512X (numérique)

Découvrir la revue

Citer cet article

Buch, E. (2012). Relire Ingarden : l'ontologie des oeuvres musicales, entre fictions et montagnes. Intersections, 32(1-2), 61-81.

https://doi.org/10.7202/1018579ar
Résumé de l'article

Qu'est-ce qu'une oeuvre musicale? La question ontologique soulevée par Roman Ingarden dans sa critique de la phénoménologie de Husserl a de nombreux points communs avec la querelle du "platonisme musical" déployée dans les années quatre-vingt par des philosophes analytiques tels que Jerrold Levinson et Peter Kivy. En 1992, Lydia Goehr a proposé une historicisation radicale du concept d'oeuvre musicale, sans pour autant liquider un projet ontologique qui, tout récemment, insiste dans les réflexions de Roger Pouivet à propos de "l'oeuvre musicale rock". Cet article fait le point sur ces débats philosophiques avant de signaler la crise sociologique de la notion d'oeuvre liée au virage numérique et à la fragmentation du répertoire classique selon les standards de l'industrie culturelle. Reprenant la définition d'Ingarden de "l'objet purement intentionnel" à partir de cet ébranlement du sens commun, il compare pour finir le statut ontologique de l'oeuvre musicale à celui des êtres de fiction, un personnage de roman par exemple, ainsi qu'à celui d'entités qui, telles les montagnes évoquées par la "théorie granulaire de la réalité" de Barry Smith, résultent de la projection d'une pratique langagière sur la surface sonore du monde.
Copyright @ Canadian University Music Society / Société de musique des universités canadiennes, 2013
Ce document est protégé par la loi sur le droit d'auteur. L'utilisation des services d'Érudit (y compris la reproduction) est assujettie à sa politique d'utilisation que vous pouvez consulter en ligne.

https://apropos.erudit.org/fr/usagers/politique-dutilisation/ 


\title{
RELIRE INGARDEN : L'ONTOLOGIE DES GEUVRES MUSICALES, ENTRE FICTIONS ET MONTAGNES
}

\author{
Esteban Buch
}

\section{QU'EST-CE QU'UNE GEUVRE MUSICALE?}

Qu'est-ce qu'une cuvre musicale? C'est le titre français du livre de Roman Ingarden, traduit de l'allemand par Dujka Smoje et publié en 1989 chez Christian Bourgois ${ }^{1}$. La forme interrogative peut sembler scandaleusement infidèle à sa source, "L'œuvre musicale», première partie de l'édition allemande de 1962 des Recherches sur l'ontologie de l'art ${ }^{2}$. Pourtant, ce changement de titre, contestable d'après les normes de l'édition scientifique, n'est pas dépourvu de fondement philologique. Le titre de la première version du texte, commencée en polonais à Paris en 1929 et complétée quatre ans plus tard à Lwow, était: «Sur l'identité de l'œuvre d'art musicale»3. Et c'est «L'œuvre musicale et le problème de son identité» qui fut retenu pour l'édition polonaise posthume parue séparément en 1973, puis traduite en anglais et en italien ${ }^{4}$. Les titres successifs vont donc de la simple désignation de l'œuvre à l'exploration d'un problème d'identité qui serait indissociable de son inscription dans l'histoire. L'interrogation de la version française a au moins le mérite de rappeler que l'œuvre musicale n'a pas le caractère d'évidence que lui attribue le sens commun.

Qu'est-ce qu'une cuvre musicale? Les tentatives de réponse incluent non seulement l'ouvrage précurseur d'Ingarden mais aussi de nombreuses contributions en philosophie analytique qui, indépendamment de lui, se sont succédées au moins depuis 1968, date de parution de Langages de l'art de Nelson Goodman et de Art and its Objects de Richard Wollheim5. De l'ensemble de ces textes, un certain consensus se dégage, qui porte avant tout sur une série de négations, déjà avancées par le philosophe polonais: l'œuvre musicale, ce n'est ni la représentation mentale qu'a le compositeur au moment d'écrire ; ni la partition manuscrite ou imprimée issue de ce processus créateur ; ni les interprétations qui en sont proposées par des musiciens; ni les enregistrements de celles-ci; pas plus que les exécutions automatiques que peuvent produire des instruments mécaniques ou informatiques; ni, enfin, les représentations dans l'esprit des auditeurs, ou dans celui des lecteurs de la partition. Ni bien entendu une combinaison quelconque de ces éléments.

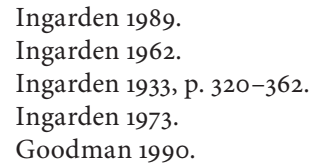


Les amateurs de mises en abyme pourront comparer ce jeu de variations négatives avec celui de l'interprétation musicale classique elle-même, fait d'écarts minimaux autour d'une partition que l'on tient pour source de la chose mais en aucun cas pour la chose elle-même. Quant à ce qu'est véritablement cette dernière, il ne faut pas écarter la possibilité qu'elle ne soit rien du tout, c'est-à-dire que la réponse la plus directe à la question: Qu'est-ce qu'une ouvre musicale? ne soit autre que: une simple idée. Cette conception sceptique n'est pas sans lien avec celle de Goodman, selon laquelle une œuvre musicale est la «classe-de-concordance» de ses interprétations, définie par la conformité de celles-ci avec la partition ${ }^{6}$. Mais plus fréquent est qu'on accorde à l'œuvre un statut ontologique plus positif, par exemple en tant que type normatif (norm-type) dont les interprétations seraient les exemples d'application (tokens), selon le vocabulaire proposé par Charles S. Peirce et repris par certains auteurs. On se rapproche ainsi d'une Idée au sens platonicien, quitte à débattre à l'infini pour savoir si ces objets idéaux sont véritablement créés ou seulement découverts?.

Qu'est-ce qu'une œuvre musicale? Dans une perspective moins philosophique que sociohistorique, on peut répondre qu'il s'agit de la catégorie fondamentale de la vie musicale occidentale de ces deux derniers siècles. C'est le constat essentiel fait par Lydia Goehr dans son livre The Imaginary Museum of Musical Works, paru en 1992, qui décrit la montée en puissance, au début du dix-neuvième siècle, du concept d'œuvre comme norme des pratiques musica$\operatorname{les}^{8}$. Ce déplacement de l'être vers le concept a une portée philosophique sur laquelle nous reviendrons. Pour l'instant, la perspective historiciste de Goehr, orientée contre ce qu'elle appelle «l'impérialisme conceptuel» de la notion d'œuvre, invite à souligner le fait que chez la plupart des auteurs - Goehr comprise d'ailleurs - l'intérêt pour le débat ontologique est né de leur fréquentation quasi exclusive du répertoire de la musique classique, traditionnellement conçu et perçu comme une série d'unités discrètes appelées opus.

Non pas qu'on ait toujours été indifférent au fait que les choses peuvent être différentes pour d'autres types de musique. Peter Kivy écrivait en 1987: «Il se peut que ce ne soit pas vrai pour les improvisations, et il se peut que ce ne soit pas vrai pour certains types de musique électronique. Il se peut que ce ne soit pas vrai là où fait défaut un système notationnel. En fait, il se peut que ce ne soit pas vrai pour la plupart des musiques de ce monde. Mais pour une bonne partie de la musique savante (art music) que l'Occident tient en plus haute estime, depuis le développement d'une notation musicale sophistiquée, il semblerait bien que ce soit vrai qu'il y a des œuvres musicales, et qu'il y a des

6 Ibid., p. 208.

7 Citons seulement, de cette importante bibliographie : Wolterstorff 1975, p. 115-142 ; Levinson 1980, p. 5-28; Kivy 1987, p. 245-251; Cox 1985, p. 367-374; Fisher 1991, p. 129-136; Davies 1991, p. 21-41. Pour un résumé critique de ses enjeux, voir Garda 2007, «Le teorie dell'opera d'arte musicale nel Novecento ", Storia dei concetti musicali. Espressione, forma, opera, sous la dir. de Gianmario Borio et Carlo Gentili, 301-310.

8 Goehr 1992. Pour une histoire du concept d'œuvre musicale depuis l'Antiquité, voir la troisième partie de Storia dei concetti musicali, op.cit. 
interprétations de ces dernières ${ }^{9} »$. Laissons de côté le ton légèrement militant de ces propos, qu'il serait facile aujourd'hui de critiquer comme une forme d'ethnocentrisme. Même formulée comme un disclaimer, la remarque de Kivy est opportune, vu la tendance dominante à réduire la diversité des pratiques musicales au problème "paradigmatique» d'une symphonie de Beethoven ou une ballade de Chopin.

Cela dit, c'est bien à l'intérieur de l'esthétique analytique - et non pas, disons, comme révolte d'un ethnomusicologue contre l'hégémonie de la musique classique -, qu’a été posée tout récemment la dépendance de la question ontologique par rapport à la pratique, autrement dit par rapport à l'histoire. C'est ce que David Davies appelle la «contrainte pragmatique» de l'ontologie: «Il ne s'agit pas seulement d'affirmer que l'épistémologie de l'art et l'ontologie de l'art exercent des contraintes l'une sur l'autre, mais que c'est bien notre pratique qui a la primauté et qui doit être le fondement de nos recherches ontologiques, car c'est elle qui détermine en général quel genre de propriétés doivent posséder les œuvres d'art ${ }^{10}$ ». Ce plaidoyer prend toute sa signification face à l'ancrage de la réflexion philosophique dans l'usage ordinaire du langage, habituel en philosophie analytique comme au sein de l'école phénoménologique, qui toutefois inclut rarement une prise en compte de la nature contingente de cet usage, résumée dans le «nous» que Davies adresse à ses pairs philosophes: our practice.

Au sein de ce «nous», au-delà du fait qu'il est mort depuis quarante ans, le nom de Roman Ingarden a tout naturellement sa place.

\section{INGARDEN ET LA QUERELLE SUR L'EXISTENCE}

L'intérêt particulier de Roman Ingarden pour l'ontologie de l'œuvre musicale s'enracine, nous y reviendrons, dans son expérience de mélomane et sa pratique de pianiste amateur ${ }^{11}$. Il n'en participe pas moins d'un projet systématique qui occupa le philosophe polonais pendant toute sa vie, celui d'une esthétique philosophique d'inspiration phénoménologique. La première mouture de son texte sur la musique est contemporain de son ouvrage sur l'œuvre d'art littéraire ; par la suite, la musique trouvera sa place au sein d'une vaste réflexion incluant également la peinture, l'architecture, le cinéma. Or le choix de se tourner vers la question de la littérature, et tout particulièrement vers la fiction, découle à son tour d'un dessein philosophique général, celui de développer une ontologie phénoménologique réaliste. Comme l'a remarqué Patricia Limido-Heulot, "cette œuvre d'apparence esthétique prend toute sa signification lorsqu'elle est replacée dans son contexte polémique dirigé contre l'idéalisme husserlien dont elle constitue, aux yeux d'Ingarden, une première réfutation ${ }^{12}$ ». La même chose peut être dite de l'essai sur la musique.

Ingarden, intégré pendant ses études doctorales au fameux cercle de Göttingen, avait dès 1918 exprimé à Husserl des réserves fondamentales à propos

9 Kivy 1987 , p. 245.

10 Davies 2009, p. 162.

11 Rieser 1971, p. 449.

12 Limido-Heulot 2001, p. 12. 
de ce qu'il tenait pour le tournant «idéaliste» du fondateur de la phénoménologie. «Il n'est alors plus besoin de parler de quelque chose qui serait essentiellement étranger à la conscience», lui écrit-il alors, sceptique, anticipant ainsi la position qu'il défendra dans son grand ouvrage La Querelle sur l'existence du monde ${ }^{13}$. Pour certains spécialistes - Mikel Dufrenne par exemple -, cette querelle repose sur un malentendu autour de la notion husserlienne d'intentionnalité ${ }^{14}$. Quoiqu'il en soit, même si cela peut évoquer surtout l'émancipation d'un disciple arrivé à maturité, la polémique ne se réduit pas à une explication en tête-à-tête avec Husserl. À plusieurs reprises, Ingarden fait mention des travaux de Waldemar Conrad, qui passe pour le premier à avoir appliqué l'approche phénoménologique au domaine de l'esthétique. Or la première partie de l'essai de Conrad Der ästhetische Gegenstand, paru entre 1908 et 1909, est consacrée à la musique, et inclut une discussion des écrits de Hugo Riemann, le musicologue allemand le plus célèbre de l'époque. L'auteur cerne cet «objet esthétique " qu'est la musique par paliers de complexité croissante, en partant de la note isolée pour se tourner ensuite vers la phrase musicale, et ainsi de suite jusqu'à l'œuvre musicale elle-même, soit l'«objet idéal» qu'est selon ses termes, par exemple, une symphonie ${ }^{15}$.

Ingarden ne songe nullement à nier l'importance historique de l'essai de Conrad en tant que "premier travail phénoménologique dans le domaine de l'esthétique». Le ton à l'égard de son collègue et précurseur reste toutefois sévère: "Il n'a rien à dire à propos des expériences esthétiques de création et de réception, et il considère les œuvres d'art (ces objets esthétiques) comme des "objets idéaux" au sens que Husserl donne à ce terme dans ses Recherches logiques, c'est-à-dire en tant qu'objets intemporels et immuables ${ }^{16}$ ». La critique des objets esthétiques conçus comme "objets idéaux" au sens husserlien est précisée dans L'ouvre d'art littéraire: «Si l'œuvre littéraire était un objet idéal, cependant, il serait impossible de concevoir qu'elle puisse commencer à exister à un moment donné et changer dans le cours de son existence, comme c'est effectivement le cas. À cet égard l'œuvre littéraire diffère radicalement de ces objectités idéales que sont, par exemple, un certain triangle, le numéro cinq, l'idée du parallélogramme, ou encore l'essence du "rouge"17». Et c'est exactement la même critique qu'Ingarden formule à propos de l'œuvre musicale:

Chaque ouvre musicale déterminée est un objet qui persiste dans le temps. Créé dans un temps donné, il existe ensuite en tant qu’œuvre spécifique, après que le processus de sa création est tout à fait terminé. C’est pourquoi contrairement à l'opinion explicite de Waldemar Conrad, il ne doit pas être considéré comme un objet «idéal». Un objet ne peut pas être en même temps idéal et créé à un moment précis par des causes réelles. On

13 Roman Ingarden 2001, p. 154.

14 Cité dans Limido-Heulot 2001, p. 69.

15 Conrad 1908, p. 77. Voir Mazzoni 1998, p. 223-233.

16 Ingarden 1975, p. 258.

17 Ingarden 1973, p. 11. Voir aussi ibid., p. 4n et p. 32n. Pour la notion d'objet idéal dans les Recherches logiques, voir notamment le $\S 8$ du chap. 2 de la Première recherche dans Husserl 1970, p. 351-2. 
ne peut mettre en doute la création d'une œuvre musicale à un moment déterminé à partir des réalités créatrices de son auteur ${ }^{18}$.

Il est frappant de reconnaître, dans cette discussion entre phénoménologues, les grandes lignes de la «querelle du platonisme musical» déployée des décennies plus tard par la philosophie analytique. Cela dit, il n'est pas sûr que Conrad fasse preuve d'une allégeance à Husserl aussi forte que celle que lui attribue Ingarden. À la fin de son essai, Conrad distingue l'objet esthétique à la fois de l'objet naturel et de l'objet géométrique, pour mettre en avant l'«état esthétique» (ästhetische Zustand) qui en caractériserait la saisie en tant qu'objet intentionnel, quitte à renvoyer à plus tard la définition exacte de cet état ${ }^{19}$. Voilà sans doute pourquoi Ingarden reproche à Conrad de négliger l'expérience esthétique - à ceci près que chez lui celle-ci reste prise dans des difficultés comparables.

En effet, voulant distinguer l'œuvre musicale autant des phénomènes acoustiques naturels que de signaux artificiels tels que le klaxon de voiture avec le leitmotif de Siegfried - courant à l'époque de ses études en Allemagne -, Ingarden explique que face à la Pathétique de Beethoven, par exemple, «nous prenons une attitude esthétique, et en la maintenant, nous appréhendons l'œuvre musicale dans sa forme propre, en tant qu'objet esthétique ${ }^{20} »$. Mais qu'est-ce qu'une attitude esthétique?

Ni l'œuvre musicale même, ni une quelconque de ses parties, n'est quelque chose d' «individuel», dans le sens où cela est valable pour chaque objet réel. Dans l'attitude purement esthétique, en partant d'une de ses exécutions, nous appréhendons, à travers les sons isolés et à travers les formations musicales de niveau supérieur, seulement les qualités pures, non individualisées par les particularités propres de l'être réel. Pendant que nous écoutons une exécution individuelle déterminée, tout en étant «branchés» sur l'œuvre même, nous faisons abstraction du mode d'existence individuel des sons et des formations sonores (de l'individualité des sons et des formes sonores que nous venons d'entendre) appartenant à l'exécution; de la réalité individuelle entendue à l'instant, nous dégageons exclusivement les qualités pures de l'œuvre sonore ${ }^{21}$.

On pourrait voir un raisonnement circulaire dans le fait de dire que l'œuvre musicale est l'objet visé par une conscience lorsqu'elle adopte l'attitude esthétique, puis de définir cette attitude par un type d'objet particulier, à savoir l'objet esthétique. À ceci près que le passage cité retient en outre une saisie des «qualités pures» de l'œuvre, au-delà du «mode d'existence individuel» propre à chacune de ses exécutions. Mais cela laisse entière la question du statut de ces qualités pures qui ne sont ici définies que par contraste avec celles des objets réels, existantes dans un temps et un espace réels.

\footnotetext{
18 Ingarden 1989, p. 51.

19 Conrad 1909, p. 455.

20 Ingarden 1989, p. 80.

21 Ibid., p. 82.
} 
En effet, une chose est sûre: l'œuvre musicale n'existe pas dans l'espace réel, du moment qu'il suffit de se déplacer dans la salle de concert pour que la perception que nous en avons en soit modifiée, sans qu'on puisse pour autant la situer dans un lieu précis. Elle n'existe pas dans un temps réel, car toutes les marques temporelles d'une expérience d'écoute concrète, qu'elles soient dues aux singularités de l'interprétation ou à notre état psychologique, sont non pertinentes pour son identité, partant pour sa définition. Et ce, sous peine de concevoir autant de Pathétique(s) de Beethoven qu'il n'y en a d'interprétations multiplié par le nombre d'auditeurs multiplié par le nombre d'auditions par chacun d'entre eux, le tout multiplié enfin, si c'est le cas, par le nombre d'exemplaires du disque concerné. Mais si l'œuvre n'est pas un objet réel existant ici et maintenant, et si elle n'est pas non plus un objet idéal existant de toute éternité, peut-on dire encore qu'elle existe? Et dans le cas affirmatif, quel est son mode d'existence? Bref, qu'est-ce qu'une ouvre musicale?

Un objet intentionnel, répond Ingarden, c'est-à-dire un objet dont l'existence est indissociable d'une conscience qui le vise. Et plus précisément, un «objet purement intentionnel ${ }^{22}$. Cette nuance est capitale. Dans la philosophie ingardienne, la notion d'objet purement intentionnel concerne les objets intentionnels dépourvus de corrélats réels. Le cas paradigmatique est celui des ficta, c'est-à-dire des représentations fictionnelles d'objets réels qu'on trouve dans les œuvres littéraires: si l'arbre est un objet réel et l'arbre-perçu-en-tant-que-tel son corrélat intentionnel, selon l'exemple classique de Husserl, l'arbre décrit dans un roman est un objet purement intentionnel. Dans cette direction, le fait que les êtres de fiction sont dépourvus de densité sémantique (nous n’avons aucune manière de savoir de quelle couleur sont les yeux d'un personnage si l'auteur a omis de le dire) est comparable au caractère schématique de la partition musicale, qui laisse à l'interprète le soin de combler les «trous» qui résultent de l'indétermination de la notation ${ }^{23}$. Aussi, les "qualités pures" saisies dans l'expérience esthétique sont elles-mêmes comparables à des fictions, du moment qu'elles ne peuvent faire l'objet que de «quasi-jugements ${ }^{24}$ ». Bref, en poussant le raisonnement jusqu'au bout nous pouvons dire que, dans la perspective ouverte par Ingarden, les œuvres musicales sont des fictions.

Nous reviendrons plus loin sur pourquoi, à notre avis, le philosophe ne fait pas explicitement sienne cette conclusion. Assurément, un tel rapprochement peut soulever des objections. Peter Kivy, par exemple, a remarqué que «les œuvres de Brahms n'habitent pas le même espace logique que les unicornes, comme le font les œuvres d'Adrian Leverkühn ${ }^{25}$ ». À cela on peut répondre que si l'on pense (contre Goodman) que la notation utilisée est dépourvue de conséquences ontologiques, alors les descriptions d'œuvres musicales dans Doktor Faustus ne différent des partitions de Brahms que par leur degré d'incomplétude, qui rend possible - et c'est bien sûr essentiel pour la pratique - une exécution des œuvres de Brahms, mais pas de celles de Leverkühn. Quoiqu'il

\footnotetext{
22 Ibid., p. 150.

23 Ibid., p. 164.

24 Voir Ingarden 2001, p. 243.

25 Kivy 1987, p. 414.
} 
en soit, même si Ingarden ne dit pas explicitement que les œuvres musicales sont comme des personnages ou des paysages de roman, il affirme bien qu'elles en partagent le statut ontologique.

Cela ne revient pas à dire que les œuvres musicales n'ont pas d'existence, moins encore qu'elles n'ont pas d'importance, vu notamment la fonction capitale de la fiction dans une perspective anthropologique ${ }^{26}$. Après tout, il est faux de dire que les unicornes n'existent pas, du moment que les arts et la littérature en regorgent, et que les gens les imaginent. En tant qu'objet purement intentionnel, l'œuvre musicale n'est nullement dépourvue de qualités. Les thèmes d'une certaine sonate se succèdent les uns aux autres de manière invariable, et cette succession n'est pas un phénomène acoustique qu'on pourrait mesurer en secondes ni même en numéros de mesure, mais relève de ce que Ingarden appelle, à la suite de Husserl, une supra-temporalité, ou encore une structure quasi temporelle immanente. Pareillement, les présentations du thème d'une fugue de Bach dans ses transpositions successives exemplifient une forme de mouvement au sein de ce qu'Ingarden appelle un espace musical pur.

Cette notion d'espace musical, qui sur le plan technique désigne essentiellement le spectre des hauteurs, contient une dimension métaphorique non explicitée, s'agissant d'une forme d'espace dépourvue d'extension, concept improbable s'il en est ${ }^{27}$. Par ailleurs, une spécificité temporelle définie tantôt comme un défaut («quasi-temporel»), tantôt comme un excès («supra-temporel»), ne se conçoit que comme un écart par rapport au temps lui-même. L'ontologie phénoménologique de la musique de Ingarden, contrairement à celle de Conrad par exemple, est indissociable de ces catégories de l'écart, comme une sorte de ligne de fuite. Dans un autre passage, Ingarden explique que «l'œuvre constitue un complément intentionnel des actes de conscience correspondants, en tant que limite idéale dont l'auteur et l'auditeur se rapprochent plus ou moins en cherchant à la concrétiser ${ }^{28}$. C'est dire littéralement le caractère borderline de son mode d'existence.

\section{FICTION, CONCEPT ET OBJET ESTHÉTIQUE INTERSUBJECTIF}

Une fiction, voilà donc ce qu'implique l'affirmation que l'œuvre musicale est un objet purement intentionnel. À ceci près que, contrairement aux fictions littéraires, dont le statut est balisé par la fameuse suspension de l'incroyance, l'œuvre musicale n'est pas reconnue comme fiction au sein des mondes de l'art correspondants. C'est là une conséquence - on pourrait dire une conséquence idéologique - du concept d'œuvre tel qu'il a circulé historiquement au sein de l'espace social. En effet, l'œuvre musicale est aussi une idée, au sens quotidien du terme, dont le statut ontique est celui des contenus de la vie psychique; une idée générique qui, sous le label d'« œuvre musicale», informe la perception et la conceptualisation des entités musicales particulières.

26 Voir Schaeffer 1999.

27 Sur la notion d'espace en musique voir notamment Osvaldo Vázquez, «El espacio en música », http://osvazqz.wordpress.com, consulté le 10 novembre 2010.

28 Ingarden 1989, p. 149. 
Or, malgré son refus de l'idéalisme de Husserl, Ingarden reste fidèle à celuici pour ce qui est de la récusation du psychologisme. Dans Qu'est-ce qu'une œuvre musicale, le deuxième chapitre est consacré à la critique de l'approche psychologique, qui envisage l'œuvre musicale seulement en tant que représentation mentale, en ciblant nommément le musicologue Ernst Kurth. C'est toutefois dans le dernier chapitre, consacré à «l'identité de l'œuvre musicale», que sont tirées les conséquences les plus intéressantes de la critique de l'approche psychologique, au moment d'élaborer la notion d'«objectités esthétiques intersubjectives ${ }^{29} »$ :

Notre conception de l'œuvre se construit au cours d'une expérience esthétique, fondée sur la façon dont une œuvre se manifeste en se concrétisant. Les résultats d'une perception directe sont réunis dans un système de concepts ou dans des théorèmes. Au lieu de référer notre point de vue à la concrétisation avec laquelle nous échangeons directement, ou à l'exécution que nous venons d'entendre, nous le rapportons sans remise en question à l'œuvre elle-même, bien que nous n'ayons pas vraiment le droit. Cependant, lorsque les conceptions de la même œuvre divergent, surtout lorsqu'elles sont établies par différents auditeurs en partant de diverses concrétisations, cela suscite souvent les disputes entre les auditeurs à propos du véritable contenu et de la valeur de l'œuvre. Par ces discussions entre les spécialistes et le grand public se constitue progressivement une vision commune, globale, de l'œuvre, correspondant alors à un objet esthétique commun, intersubjectif, d'ordre supérieur. C'est un complément intentionnel de l'opinion, non d'un seul, mais d'une communauté d'auditeurs dans un pays et dans un temps déterminéz ${ }^{\circ}$.

Dans le report des traits perçus d'un phénomène sonore vers ceux de l'œuvre musicale elle-même s'effectue sans en avoir vraiment le droit - le droit au sens logique s'entend -, nous pouvons voir une autre formulation de l'idée que l'œuvre musicale est proche d'une fiction qui s'ignore. Avec cette ouverture sur l'intersubjectivité, toutefois, Ingarden définit avec précision, quoique peut-être involontairement, l'espace d'une approche sociologique de l'ontologie musicale. La constitution progressive et contradictoire d'une "vision commune» de l'œuvre, conçue par la même occasion comme «objet esthétique intersubjectif»; une vision commune jamais complètement stabilisée au long de l'histoire, et pourtant agissante tout le long en tant qu'idée régulatrice, voilà qui constitue une bonne description d'une certaine dynamique sociale des échanges discursifs à propos de la musique. À moins qu'il ne faille dire, plutôt, "écoute commune»: basée sur des pratiques musicales réelles, l'œuvre musicale devient ainsi, pour résumer, une fiction qui s'ignore, stabilisée dans une écoute commune par l'échange discursif.

Or, si l'intersubjectivité occupe ainsi un rôle clé dans l'ontologie d'Ingarden, proche en cela d'une certaine herméneutique ${ }^{31}$, ce n'est certes pas une fiction

\footnotetext{
29 Ibid., p. 181, traduction modifiée.

30 Ibid., p. 180.

31 Voir Garda 2007, p. 300-301.
} 
qui en constitue l'objet déclaré. Autrement dit, Ingarden ne songe pas du tout à proposer une critique de l'idéologie, fût-ce celle de l'idéologie dominante dans la vie musicale. Cela ne s'explique pas seulement par sa distance vis-à-vis du marxisme. Souvent son discours sur la musique semble étrangement familier, ou plutôt accordé avec le sens commun de la tradition classique. Il rejoint à bien d'égards cette esthétique musicale pratique qui, sous forme de critiques journalistiques ou d'ouvrages de vulgarisation musicologique, de propos de compositeurs ou de musiciens, de conversations de connaisseurs ou d'habitués, a fourni les bases d'une casuistique du jugement, inséparable des institutions de la musique classique depuis les débuts du dix-neuvième siècle. Cela rejoint à nouveau la démarche husserlienne, qui ancre la réflexion philosophique dans les opinions «pré-scientifiques ${ }^{32}$ ». À ceci près que la «science» est ici une ontologie appliquée dont l'objet échappe, par définition, à l'aire de compétences spécifique de l'auteur. C'est pourquoi les propos d'Ingarden sur l'œuvre musicale peuvent être vus comme l'élaboration rigoureuse, faite par un philosophe professionnel, de notions courantes tirées de sa pratique amateur de musicien et de mélomane, selon un périmètre d'intérêts dominé par les noms de Beethoven et Chopin.

Cela renvoie à la nature des compétences du philosophe polonais, à l'évidence non négligeables, en matière d'histoire et d'analyse de la musique. Concentrons-nous sur sa perplexité face à une question apparemment mineure, celle de savoir si les silences qui séparent les différents mouvements d'une œuvre par exemple une sonate, d'habitude en quatre mouvements - font partie ou non de celle-ci. Sa réponse est négative, vu que ce silence peut durer plus ou moins longtemps, que les gens en profitent pour tousser ou pour parler à leur voisin, etc. C'est très différent des silences à l'intérieur d'un même morceau de musique, qui relèvent d'un phénomène musical à part entière, comparable aux sons qui le précèdent et le suivent. Cependant, remarque-t-il, on ne saurait mettre en doute le fait que la sonate en question consiste de ses quatre mouvements, et que l'ordre prescrit est décisif pour son identité. Qui plus est, l'écoute du deuxième mouvement doit avoir lieu sous l'emprise de l'écoute du premier, ce qui implique que ce silence ne soit pas trop long, etc. Dans ces conditions, dit Ingarden, «ces "interruptions" sont maintenant quelque chose qui relie les parties isolées entre elles, et forge une entité cohérente en quatre parties ${ }^{33}$ ».

Cela dit, comment savons-nous, au juste, que chacune de ces interruptions n'a pas marqué la frontière entre une œuvre et une autre œuvre, mais bien entre deux parties de la même œuvre? Qu'est-ce qui fait que les quatre mouvements de la Cinquième symphonie de Beethoven constituent bien une œuvre, tandis que ce n'est pas le cas avec la structure supra-temporelle que forment-c'est l'exemple d'Ingarden- Nuages de Debussy suivi d'une Tocatta de Bach puis d'une aria de Madame Butterfly de Puccini? "Le manque de cohérence d'une telle construction nous sautera aux yeux", répond le philosophe,

32 Voir par exemple Husserl [1931] 1992, p. 32-39.

33 Ingarden 1989, p. 161. 
en se réclamant ainsi du sens commun ${ }^{34}$. Sans doute, mais encore? Qu'est-ce qui empêcherait de dire qu'il s'agit d'une œuvre musicale mauvaise car incohérente, sans pour autant cesser de voir un objet appartenant à la catégorie «œuvre musicale»? Et comment cerner précisément la cohérence, et le manque de cohérence?

Cela montre bien la dimension normative que revêt le concept d'œuvre musicale, même au sein d'un texte qui, comme celui d'Ingarden, évite scrupuleusement les jugements de valeur. Or c'est précisément à ce stade que le philosophe renvoie au musicologue la tâche d'établir les liens entre les différents mouvements:

Tout comme les différentes formes de genre "sonore» peuvent établir la base de l'unité et de l'intégrité des œuvres musicales plus courtes (par exemple les préludes), ou des mouvements séparés des œuvres plus vastes (sonate, symphonie), dépendant de leur construction plus ou moins complexe, plus intégrée ou détachée, les formes musicales d'ordre supérieur représentent aussi le moment unificateur grâce auquel tous les motifs font partie d'une seule et même œuvre, que ce soit par des qualités émotives particulières, ou bien par des manifestations «harmonieuses» (ou contrastantes). Ainsi nous avons trouvé là le principe qui nous permet de comprendre de quelle façon une œuvre contenant beaucoup de parties disparates, et qui se développe dans le temps musical, peut pourtant être une œuvre et une totalité. [...] Ce principe montre la direction dans laquelle on peut chercher l'unité et l'intégrité interne d'une œuvre, sans montrer nécessairement la raison spécifique de son unité. Cette raison - ici déterminée seulement d'après le type de base - doit être explorée séparément dans chaque cas. Toutefois, ce n'est plus la tâche de la théorie philosophique générale, mais celle de la recherche musicologique spécialisée 35 .

Tout cela fait signe vers le contexte historique de la proposition d'Ingarden. Le philosophe présuppose une certaine cohérence motivique et thématique censée constituer une totalité sans laquelle, à proprement parler, il n'y a pas d'œuvre musicale du tout. C'est cette cohérence, dite organique, que depuis la deuxième moitié du dix-neuvième siècle des générations de musicologues ont cherché à identifier au moyen de l'analyse musicale, quitte à parfois forcer le trait pour établir des connexions entre des éléments fort dissemblables. Et peu importe si en toute rigueur, n'en déplaise aux tenants de l'organicisme, même la plus obsessionnelle des récurrences motiviques au long des différents mouvements n'est pas suffisante pour statuer sur l'unité de l'œuvre, du moment qu'il est toujours possible d'y voir des entités distinctes et indépendantes, quoique basées sur le même répertoire de motifs. La Cinquième de Beethoven occupe à cet égard chez tous les théoriciens une place paradigmatique ${ }^{36}$. Ce n'est pas un hasard si elle réapparaît dans le texte d'Ingarden, qui montre ainsi sa dette avec la musicologie de son temps.

\footnotetext{
34 Ibid., p. 162.

35 Ibid, p. 157-158.

36 Voir notamment Burnham 1995.
} 
Le philosophe postule l'existence d'une catégorie générique d'objets, l'œuvre musicale, tout en se déclarant incompétent pour statuer sur l'appartenance à ce genre des individus qu'il rencontre dans le monde phénoménal, si ce n'est au nom du sens commun: "Cela saute aux yeux». Au musicologue revient de décrire avec rigueur ce que d'un côté l'intuition propre au moment de l'écoute, de l'autre la philosophie, ne peuvent cerner que de manière confuse ou générale. C’est bien lui qui, seul compétent pour saisir la «raison spécifique» de l'unité de l'œuvre, fait autorité dans ce domaine.

Voilà une division du travail qui ne posait pas de problème particulier aussi longtemps que la science, incarnée dans ce cas par le musicologue, et la doxa, qui inclut ici le discours du philosophe dans sa condition de mélomane, s'accordaient sur l'essentiel, à savoir la centralité de la catégorie d'œuvre pour l'expérience esthétique comme pour le travail d'analyse qui en explicite l'ordre structurel à partir des postulats de cohérence et d'unité. Or, voilà qui pourrait n'être plus tellement le cas aujourd'hui.

\section{LA CRISE DE L'GeUVRe MUSICALE, ENTRE DUChAMP ET L' ITUNES STORE}

La publication du livre de Lydia Goehr en 1992 peut être vue non seulement comme un apport majeur à la discussion sur le concept d'œuvre musicale, mais aussi comme un indice de la crise de ce dernier. Sa réflexion historiciste, proche de la new musicology alors en plein essor, montre qu'il y a une vingtaine d'années on n'en était plus à l'âge de la construction du «musée imaginaire des œuvres musicales", mais bien à celui de sa déconstruction. L'aphorisme de Theodor W. Adorno «le tout est le faux», qui en musique résonne comme critique de l'œuvre traditionnelle, devient à cette époque une intuition largement partagée dans les études sur les arts, musique comprise ${ }^{37}$.

Or tout porte à croire qu'en vingt ans cette crise n'a fait que s'accentuer, et ce non seulement à cause du cours pris par la réflexion musicologique et philosophique, mais bien des modifications de la pratique: d'une part, avec la dissémination du geste critique des avant-gardes, qui a fait reculer l'importance de l'œuvre au profit de la conception de «l'art comme performance», présente surtout dans les arts visuels, mais non dépourvue d'effets dans le domaine musical ; d'autre part, avec la révolution numérique et sa transformation des modalités de la consommation, qui incluent désormais une commercialisation et une diffusion fragmentée des œuvres classiques en accord avec les formats typiques de la musique de masse ; enfin, avec l'érosion de la hiérarchie entre les genres musicaux, qui remet en question la dimension normative de cette musique classique où la notion d'œuvre avait pris son allure conceptuelle et sa portée prescriptive. Cela nous amène à envisager en retour une modification des habitus et des habitudes des élites intellectuelles, où s'enracinent les propositions théoriques sur l'ontologie de l'œuvre.

37 Nous ne pouvons évoquer ici la question complexe de l'œuvre musicale chez Adorno, que résumait la phrase : «Les seules œuvres qui comptent aujourd'hui sont celles qui ne sont plus des “œuvres" ». (Adorno 1962, p. 42). Voir notamment Garda 2007, p. 312-316. 
De ces trois niveaux du phénomène d'érosion de la catégorie d'œuvre, celui des avant-gardes est le plus ancien, et aussi le plus ambigu. En fait, dès les débuts du vingtième siècle on voit apparaître des compositions qui, comme Erratum musical I et Erratum musical II de Marcel Duchamp (1913), n'exigent pas de réalisation sonore du tout, justifiant l'expression de «musique conceptuelle» récemment proposée par Sophie Stévance ${ }^{38}$. Le geste de Duchamp a assurément une portée ontologique, du moment qu'il introduit une nouveauté spectaculaire dans le type d'objet intentionnel qui en résulte. Ce faisant, toutefois, le créateur de La roue de bicyclette est loin de congédier la notion d'œuvre elle-même, qu'au contraire il étend à toute une série d'objets aux corrélats réels incertains. Et la même chose peut être dite des productions de John Cage qui, comme la célèbre "œuvre silencieuse» 4'33, ont laissé leur empreinte sur l'histoire de la musique. Comme l'a remarqué Goehr, loin de conduire à l'abandon de la catégorie d'œuvre, «les “œuvres” de Cage ont survécu et sont même devenues représentatives du répertoire des œuvres musicales de l'avant-garde 39 ».

C'est pourquoi Goehr ne partage pas le diagnostic établi par Carl Dahlhaus qui, en 1971, identifiait une « désagrégation du concept d'œuvre musicale» dans la vogue de l'improvisation, ou encore dans Momente de Stockhausen, basée sur une idée de la forme-moment (Momentform) qu'il tenait pour contradictoire avec la véritable notion de forme, conçue en termes de structure ${ }^{40}$. Il faut dire que rétrospectivement cette tentative de sauvetage d'une idée d'œuvre décrite par Dahlhaus comme "dominante et pourtant impuissante», et sans laquelle il ne saurait y avoir d'art à proprement parler, paraît singulièrement dogmatique ${ }^{41}$. En tout cas, toutes ces pratiques avant-gardistes, si elles n'ont nullement conduit à l'abandon de la catégorie d'œuvre, ont incontestablement élargi le spectre de ses applications, tout en aggravant ce que le musicologue appelait son caractère "précaire». La fortune de l'« œuvre ouverte» théorisée par Umberto Eco est à cet égard exemplaire ${ }^{42}$.

Pour leur part, les évolutions technologiques introduisent souvent des modifications substantielles de la pratique dont on peine à saisir immédiatement toutes les implications philosophiques. Sans remonter jusqu'à Walter Benjamin, signalons que Ingarden constatait déjà que l'apparition du disque et autres procédés mécaniques de reproduction était de nature à avoir des conséquences ontologiques, à propos desquelles il restait toutefois dubitatif: "Remarquons qu'actuellement il n'est pas nécessaire de "noter" les œuvres musicales à l'aide d'un moyen aussi imparfait que la notation musicale. Il serait possible d'utiliser pour cela les appareils modernes qui fixent l'œuvre sur disque, ou bien grâce au système des ombres, la noter dans tous ses détails sur une pellicule, et ainsi lui donner une existence durable, rendant possible des exécutions identiques de l'œuvre ; d'autre part, cela lui assurerait un mode d'existence réel ${ }^{43} »$. Cette

\footnotetext{
38 Voir Stévance 2009.

39 Goehr 1992, p. 265.

40 Dahlhaus 1971, p. 142. Voir Goehr 1992, p. 269 n.

41 Ibid., p. 136.

42 Voir notamment De Benedictis 2007, p. 317-334.

43 Ingarden 1989, p. 148.
} 
ouverture sur un mode d'existence non intentionnel est toutefois immédiatement contredite: "L'appareil sur lequel tourne le disque avec l'œuvre "notée" ne peut réellement rien produire d'autre qu'un système déterminé d'ondes sonores. Lorsque ni l'auteur ni quelqu'un d'autre ne reçoit ces ondes, l'œuvre ne peut se manifester dans sa forme phénoménale et qualitativement déterminée ; en réalité il n'y a pas d'exécution de l'œuvre musicale ${ }^{44}$ ».

Rétrospectivement, il semble clair que le disque, loin de modifier le découpage habituel du répertoire classique en œuvres, a plutôt eu tendance à en confirmer la pertinence, ne serait-ce qu'en établissant l'équivalence, étrangère au rituel du concert, entre une œuvre, un produit, un acte d'achat et un moment d'écoute. Bien entendu, un disque peut contenir plus d'une œuvre, et par ailleurs les éditions discographiques de «morceaux choisis» ont joué une fonction non négligeable dans la diffusion du répertoire classique. Mais tout porte à croire qu'au vingtième siècle le disque est devenu le meilleur garant de l'unicité de l'œuvre musicale classique.

Les choses sont un peu différentes avec la radio. La diffusion des classiques y oscille traditionnellement entre la reproduction du format du concert, où plusieurs œuvres se succèdent au sein d'un même moment d'écoute, et la présentation de fragments isolés pour des raisons qui, souvent, sont étrangères à toute notion d'unité syntagmatique, comme dans nombre d'émissions didactiques sur l'histoire de la musique. Le dispositif de radiodiffusion fait toutefois volontiers du fragment une sorte de synecdoque de l'œuvre entière, qui conserve son statut d'idée régulatrice tout en disparaissant - et ce n'est certes pas négligeable - du niveau phénoménal.

Or le changement est plus significatif dès lors que le principe même de la programmation radiophonique devient la mise en série de fragments. C'est un modèle de l'enchaînement des unités qui n'est plus gouverné par l'idée de clôture de l'œuvre musicale classique, mais bien par celui d'une série a priori ouverte de morceaux qui en général ne renvoient pas à un niveau syntagmatique supérieur. On a le droit de trouver cela consternant, à la suite d'Adorno qui dès 1938 diagnostiquait, dans la fragmentation des classiques, un symptôme de la «régression de l'audition ", ou bien d'y voir, surtout, un changement de perspective ${ }^{45}$. Quoiqu'il en soit, en France cette pratique est celle de Radio Classique, qui diffuse régulièrement des séquences de mouvements isolés d'œuvres classiques, lesquels par un heureux hasard ont des durées comparables aux quelques minutes qui sont la norme pour les variétés et autres musiques populaires ${ }^{46}$. Cela est fait avec des logiciels qui proposent ces séquences en fonction de critères tels que la popularité, la fréquence de diffusion, le tempo, la durée, etc. Voici par exemple, la programmation du 8 novembre 2010 entre 12 et $13 \mathrm{~h}$, telle qu'elle est présentée sur le site de la station 47 :

44 Ibid., p. 149.

45 Adorno 2001.

46 Voir Emmanuel Berretta 16 novembre 2007 et 16 avril 2008, sur www.lepoint.fr (consulté le 8 novembre 2010).

47 Cf. http://www.radioclassique.fr/ (consulté le 8 novembre 2010). 
12 ho2 Offenbach Jacques, Fille du Tambour Major (La): chanson de la fille du tambour major

12 ho4 Haydn Joseph, Quatuor à cordes "Le Cavalier": Finale "Allegro con brio" (a donné le surnom)

12 h13 Schumann Robert, Symphonie $n^{\circ} 4$ : 1er mvt

12h25 Chopin Frédéric, Étude op. $25 \mathrm{n}^{\mathrm{O}} 1$

$12 \mathrm{~h} 27$ Chopin Frédéric, Étude op. $25 \mathrm{n}^{\mathrm{O}} 3$

12 h29 Rossini Gioacchino, Concerto pour basson: Finale "Rondo"

12h34 Chabrier Emmanuel, Bourrée fantasque

12h44 Rachmaninov Serge, Étude-Tableau op. $39 \mathrm{n}^{\circ} 5$

12h49 Dvorak Antonin, Symphonie no 9 "du Nouveau Monde": Finale

La révolution numérique affecte de manière encore plus décisive les modalités de commercialisation de la musique classique. Le format $\mathrm{mp} 3$ a banalisé l'échange légal et illégal de fichiers qui, là encore, correspondent à ce format standard de quelques minutes. Or dans ce système un mouvement isolé d'une sonate ou d'une symphonie peut avoir une circulation indépendante de celle d'autres parties de la même ouvre. Par exemple, sur un site peer to peer on a plus de chances de trouver la marche funèbre de l'Hérö̈que de Beethoven que la symphonie tout entière. Pour sa part, le site iTune Store propose une vente en ligne séparée de chacun des mouvements à un prix uniforme de 99 centimes d'euro la pièce, qui est d'ailleurs le même que pour les chansons ou les morceaux d'autres types de musique. Il en résulte une standardisation de l'offre basée sur l'équivalence relative entre le prix, la durée, le nombre de bits et le genre, qui tend à dissoudre les liens privilégiés entre les fragments d'une même œuvre classique. Sous la forme stabilisée d'une liste de lecture, il devient possible d'isoler un morceau du reste, d'inverser l'ordre des mouvements, ou de les inclure dans une combinaison quelconque d'autres unités du répertoire, ou encore d'unités d’autres répertoires génériques.

Ce système permet de tirer toutes les conséquences d'un fait bien connu des mélomanes, à savoir la capacité variable qu'a une partie de l'œuvre classique pour représenter le tout: alors que tout le monde, ou presque, peut reconnaître la Cinquième de Beethoven à partir de son premier mouvement, et même des deux premières mesures de ce mouvement, cette capacité est bien moindre s'agissant, mettons, du troisième. Précisons toutefois que l'œuvre reste alors présente dans le titre, qui continue à orienter par exemple les recherches par mots-clés. Ainsi, rien n'interdit les listes de lectures telles que: Beethoven, Cinquième symphonie, premier mouvement ; Beethoven, Cinquième symphonie, deuxième mouvement... et ainsi de suite. Il est probable quaussi longtemps que la musique classique sera socialisée comme un genre distinct, la notion d'œuvre continuera à exister. Mais, dans les actuelles conditions techniques et sociologiques, le déclin de son rôle pratique comme unité temporelle de référence ne peut que s'amplifier. 


\section{GENRES MUSICAUX, OBJETS ET STRUCTURE GRANULAIRE DE LA RÉALITÉ}

L'érosion de la centralité de la musique classique pour le système des genres introduit un troisième facteur de déstabilisation de la catégorie d'œuvre. Dans le livre de Goodman, Langages de l'art, le mot «musique» ne désigne jamais que ce qui peut être saisi par un «système notationnel» défini d'après le modèle classique ${ }^{48}$. Dans le texte de Kivy cité plus haut, la reconnaissance du fait que l'existence d'œuvres musicales pourrait être mis en doute pour «la plupart des musiques de ce monde» n'est jamais qu'une concession. Depuis cette époque, la montée de la consommation "omnivore», autrement dit l'éclectisme générique, n'a fait que se confirmer au sein des élites des pays occidentaux - quand ce n'est pas le rock qui est passé en tête de leurs préférences, avec à terme la reconstitution possible d'une attitude «univore» par rapport à ce seul genre49. Dans ces conditions, il devient de plus en plus improbable qu'un philosophe, par exemple, veuille tirer des conséquences générales sur la musique à partir de l'examen du seul corpus classique.

De ce moment historique, l'ouvrage de Roger Pouivet Philosophie du rock, paru en 2010, témoigne avec éclat, quoique d'une manière paradoxale $5^{\circ}$. L'auteur y développe une ontologie de l'œuvre musicale à partir de ce genre rock qui, indice supplémentaire de sa légitimation récente, était jusque-là pratiquement absent de toute la bibliographie philosophique sur la question. Sa thèse est la nouveauté ontologique de ce qu'il appelle «l'œuvre musicale rock», une nouveauté qui serait due non pas à un quelconque trait stylistique ou sociologique mais au fait que, selon lui, les disques de rock ont été les premiers artefacts culturels de l'art de masse à être conçus et réalisés comme des «œuvres phonographiques ${ }^{51}$ ». Pour Pouivet, en effet, un disque des Grateful Dead - ses musiciens favoris - n'est pas l'enregistrement d'une œuvre musicale des Grateful Dead, moins encore l'enregistrement d'un ensemble d'œuvres ou de morceaux ou de chansons composées par ce groupe, mais bien cette œuvre musicale elle-même.

Voilà qui replace l'ontologie de la musique dans une "ontologie du sens commun» focalisée sur des objets réels, très loin à la fois du platonisme musical et de l'approche ingardienne en termes d'objet intentionnel. «[L]e cas des œuvres musicales rock ne me semble en rien ontologiquement différent de ceux des chapeaux, des bateaux, des vélos, des cachets d'aspirine, des aéroports, et d'autres artefacts ${ }^{52} »$. Cela revient à élargir la pertinence heuristique de cette notion d'œuvre qui, courante en musique classique, est historiquement étrangère au monde du rock. En d'autres termes, cela implique la décision d'appliquer la catégorie d'œuvre à des objets musicaux qui jusque-là existaient très

48 Voir Goodman 1990, p. 218ss.

49 Voir Peterson et Kern 1996, p. 900-907; Sullivan et Katz-Gerro 2007, p. 123-137 ; et Donnat 2009, p. 135-138.

50 Pouivet 2010.

51 Ibid., p. 54. Ce n'est pas le lieu de discuter de la validité de cette thèse pour l'histoire du rock. Voir Guesdon 2010, p. 269-278.

52 Ibid., p. 113. 
bien sans elle. Ce faisant, on peut penser que l'auteur transpose au monde du rock, de manière implicite et peut-être abusive, les exigences formelles traditionnellement associées à l'œuvre musicale classique, telles que l'unicité, la cohérence ou la complétude.

En fait, Pouivet n'est pas le premier à utiliser le concept d'œuvre pour parler du rock. Comme le résume Jan Butler, ces dernières années la question a divisé les spécialistes du genre, entre ceux qui - tel Richard Middleton - récusent absolument ce terme, en y voyant une forme de «réification», et ceux qui l'utilisent volontiers pour désigner, selon les termes de Philip Tagg, « un continuum musical d'une durée déterminée dont la structure interne est suffisamment cohérente pour qu'il soit identifiable en soi par ses sonorités et distinct de tout ce qui le précède et le suit, ainsi que de tout autre groupement de séquences de sons musicaux ${ }^{53} »$. Ainsi, sans reprendre à son compte les contraintes organicistes de l'œuvre classique, Tagg conserve néanmoins la notion de cohérence interne. Et ce, contrairement à Pouivet qui, lui, définit l'œuvre seulement comme "ce qui est fait par une personne ou un groupe de personnes, avec l'intention de parvenir à une entité qui, sans être ontologiquement indépendante, possède des propriétés qui en font une certaine chose». L'œuvre n'est jamais qu'un synonyme d'un «produit musical identifiable» dont on doit pouvoir constater la stabilité ontologique en disant: c'est la même œuvre. Autrement dit, il s'agit d'« un concept d'identité numérique au sujet des produits musicaux». Dans ces conditions, affirme Pouivet, la catégorie d'œuvre est «métahistorique ${ }^{54}$ ».

Avec une telle définition, en effet, il devient difficile de nier sa pertinence quasi universelle. Mais en retour pour cette extension il semble bien qu'il faille renoncer à distinguer, au sein des pratiques musicales, des entités pourvues d'une quelconque spécificité, du moment que tout objet limité dans le temps voire peut-être ceux dépourvus a priori de limites temporelles - peut en principe appartenir à cette catégorie. Ainsi, la Cinquième de Beethoven est une œuvre, tout comme Sgt Pepper's des Beatles; mais l'Andante con moto de la Cinquième et Lucy in the Skies With Diamonds en sont aussi ; voire tel ou tel passage d'un de ces deux disques, le motif initial de la Cinquième par exemple, ou encore l'accord final de A Day in the Life. Étant entendu, bien sûr, que pour Pouivet ce n'est pas le disque en tant qu'objet physique (ni le CD, ni la série de fichiers mp3 contenant l'ensemble des morceaux du 33 tours original...) qui constitue l'objet «réel» qu'est l'œuvre musicale rock, mais bien l'enregistrement qu'il permet d'entendre.

Pouivet se livre à une critique frontale de ce qu'il appelle (en réponse au reproche sur l'impérialisme conceptuel de la notion d'œuvre) «l'impérialisme historiciste» de Lydia Goehr, dû essentiellement, selon lui, à «une confusion entre une chose et son concept ${ }^{55}$ ». Or, il est bien sûr fâcheux de confondre une chose et son concept; cela n'empêche pas de penser que les concepts jouent un rôle dans la perception et la socialisation des choses, et que le rapport entre la

\footnotetext{
53 Philip Tagg 200o; cité dans Butler 2010, p. 43.

54 Pouivet 2010, p. 52.

55 Ibid., p. 181.
} 
chose et le concept n'est pas de ceux qu'un chercheur isolé peut modifier, tout au moins sans s'exposer à de graves malentendus. C'est là, précisément, tout l'intérêt de saisir ce rapport dans sa dynamique sociohistorique, en l'occurrence focalisée sur l'interrogation: Qu'est-ce qu'une cuvre musicale?

Reste à se demander: Qu'est-ce qu'une chose? Ou plutôt, pour contourner la résonance heideggérienne: Qu'est-ce qu'un objet? Renvoyons ici, comme début de réponse, à la théorie de la «structure granulaire de la réalité56». La notion d'objet peut s'appliquer à tous les niveaux où «grains» qui constituent le réel, depuis la physique subatomique jusqu'à la cosmologie, et c'est l'épistémologie qui, dans ces cas, décide de sa pertinence. Au niveau des "partitions de granularité intermédiaire» où se déroule la vie humaine, toutefois, c'est surtout au niveau de la pratique qu'en est réglé l'usage57. Très souvent cela ne pose aucun problème, car l'essentiel de nos activités se passe au contact d'entités aux frontières stables et définies, par exemple les organismes vivants tels que les personnes ou les animaux, ou les artefacts d'usage quotidien tels que les chaises ou les chapeaux. Mais c'est déjà plus compliqué avec, disons, les montagnes, que nous prenons spontanément pour des objets malgré le fait qu'il est impossible d'en identifier les limites précises (sauf éventuellement le sommet). Au point qu'on peut se demander si les montagnes - l'Everest par exemple existent "vraiment», au-delà du fait que nous parlons comme si elles existaient. Voici la réponse de Barry Smith et David Mark: «L'Everest est le résultat de la projection de ces pratiques langagières sur la surface de la Terre, de manière à découper un certain territoire possédant une certaine forme et une certaine constitution matérielle ${ }^{58}$ ».

Bien des différences existent entre une montagne et une œuvre musicale. Mais, pour ce qui est de leur constitution en objets, la comparaison n'est pas tout à fait absurde, entre la surface terrestre et l'ensemble de ce qu'on entend sous le nom de musique aux quatre coins du globe. Les contraintes pragmatiques à l'intérieur de chaque culture sont éminemment historiques, et ainsi en est-il du signifiant «œuvre musicale», qui peut s'appliquer à un vaste spectre de phénomènes. Faut-il seulement retenir, pour décrire une pratique musicale quelconque, une segmentation temporelle composée d'objets qu'on aligne comme des individus ontiques? Et dans le cas affirmatif, la notion d'œuvre reste-t-elle intéressante pour en désigner certains? Et alors faut-il la prendre, comme Pouivet, comme un simple synonyme de chose, ou bien y voir plutôt, à la suite de Ingarden, une forme sophistiquée de fiction? Peut-être vaut-il mieux tâcher de décrire comment le mot rencontre la chose, et ce que cela fait à l'écoute des êtres humains.

[Édition originale de ce texte: Buch, Esteban. 2013. «Relire Ingarden: l'ontologie des œuvres musicales, entre fictions et montagnes», Roman Ingarden:

56 Voir Bittner et Smith 2003, http://ontology.buffalo.edu/smith/articles (consulté le 10 novembre 2010).

57 Nef 2006, p. 181.

58 Smith et Mark (sans date), p. 14, http://ontology.buffalo.edu/smith/article (consulté le 10 novembre 2010). 
ontologie, esthétique, fiction, sous la dir. de Christophe Potocki et Jean-Marie Schaeffer, 177-194. Paris: Éditions des Archives Contemporaines. ]

\section{RÉFÉRENCES}

Adorno, Theodor W. 2001. Le caractère fétiche dans la musique et la régression de l'audition. Paris: Alia.

1962. Philosophie de la nouvelle musique. Paris: Gallimard/Folio.

Berretta, Emmanuel. 2008. «France Musique repasse devant Radio Classique», Le Point, 16 avril. www.lepoint.fr ( consulté le 8 novembre 2010).

.2007. «Radio Classique: Hit classic only», Le Point, 16 novembre. www. lepoint.fr (consulté le 8 novembre 2010).

Bittner, Thomas et Barry Smith. 2003. "A Theory of Granular Partitions». http://ontology.buffalo.edu/smith/articles ( consulté le 1o novembre 2010).

Burnham, Scott. 1995. Beethoven Hero. Princeton: Princeton University Press.

Butler, Jan. 2010. «Euvres musicales, reprises et Strange Little Girls», Volume! 7/1: 43.

Conrad, Waldemar. 1908. «Der ästhetische Gegenstand. Eine phänomenologische Studie» (première partie), Zeitschrift für Ästhetik und allgemeine Kunstwissenschaft 3. Stuttgart: F. Enke.

— 1909. «Der ästhetische Gegenstand. Eine phänomenologische Studie» (troisième partie), Zeitschrift für Ästhetik und allgemeine Kunstwissenschaft 4. Stuttgart: F. Enke.

Cox, Renée. 1985. «Are Musical Works Discovered? », The Journal of Aesthetics and Art Criticism 43/4:367-374.

Dahlhaus, Carl. 1971. "La désagrégation du concept d'œuvre musicale». Essais sur la nouvelle musique. Genève: Contrechamps.

Davies, David. 2009. "The Primacy of Practice in the Ontology of Art», The Journal of Aesthetics and Art Criticism 67/2:162.

Davies, Stephen. mars 1991.« The Ontology of Musical Works and the Authenticity of their Performances", Noûs 25/1: 21-41.

De Benedictis, Angela Ida. 2007. «Opera aperta: teoria e prassi», Storia dei concetti musicali. Espressione, forma, opera, sous la dir. de Gianmario Borio et Carlo Gentili. 317-334. Roma: Carocci.

Donnat, Olivier. 2009. Les Pratiques culturelles des Français à l'ère numérique. Paris: La Découverte - Ministère de la Culture et de la Communication.

Fisher, John Andrew. printemps 1991.«Discovery, Creation, and Musical Works ", The Journal of Aesthetics and Art Criticism 49/2: 129-136.

Garda, Michela. 2007. «Le teorie dell'opera d'arte musicale nel Novecento». Storia dei concetti musicali. Espressione, forma, opera, sous la dir. de Gianmario Borio et Carlo Gentili, 301-310. Roma: Carocci.

Goehr, Lydia. 1992. The Imaginary Museum of Musical Works: An Essay in the Philosophy of Music. Oxford: Clarendon Press.

Goodman, Nelson. 1990. Langages de l'art. Nîmes: Éditions Jacqueline Chambon.

Guesdon, Maël. 2010 . «L'ontologie du rock de Roger Pouivet», Volume! 7/1 'La reprise': 269-278. 
Husserl, Edmund. [1931] 1992. Méditations cartésiennes. Paris: Vrin.

-1970. Logical Investigations Vol. 1, trad. Par J.N. Findlay. London: Routledge et Paul Kegan. Réimpression avec la permission de Taylor et Francis Books Ldt.

Ingarden, Roman. 2001. "Lettre à Husserl sur la 6e Recherche logique et l'idéalisme», Husserl, la controverse Idéalisme - Réalisme, textes introduits, traduits et commentés par Patricia Limido-Heulot, 154. Paris: Vrin.

- 2001. "Qu'y a-t-il de nouveau dans la Krisis de Husserl? », Husserl, la controverse Idéalisme - Réalisme, textes introduits, traduits et commentés par Patricia Limido-Heulot, 243. Paris: Vrin.

1989. Qu'est-ce qu'une œuvre musicale, trad. Dujka Smoje.

Paris: Christian Bourgois,

— printemps 1975. "Phenomenological Aesthetics: An Attempt at Defining Its Range», The Journal of Aesthetics and Art Criticism 33/3:257-269.

-1973. Utwór muzyczny i sprawa jego tozsamosci, Cracovie, Polskie Wydawnictwo Muzyczne, ; The Work of Music and the Problem of Its Identity, trad. par Adam Czerniawski, sous la dir. de Jean Harrell. Berkeley et Londres: University of Califomia Press et Macmillan, 1986 ; L'opera musicale e il problema della sua identità. Palermo: Flaccovio, 1989.

. 1973. The Literary Work of Art. Evanston: Northwestern University Press.

-1962. «Das Musikwerk», Untersuchungen zur Ontologie der Kunst: Musikwerk, Bild, Architektur, Film. Tübingen: Niemeyer. Trad. anglaise, The Ontology of the Work of Art: The Musical Work, the Picture, the Architectural Work, the Film, traduit par Raymond Meyer et John T. Goldthwait. Athens: Ohio University Press, 1989.

_.1933. Zagadnienie tozsamosci dziela muzycznego », Przeglad Filozoficzny $36 / 4: 320-362$

Kivy, Peter. Juillet 1987. «Platonism in Music: Another Kind of Defense», American Philosohical Quarterly 24/3: 245-251.

- Été 1987. [Compte-rendu de l'ouvrage d'Ingarden], The Journal of Aesthetics and Art Criticism 45/4: 413-415.

Levinson, Jerrold . Janvier 1980. "What a Musical Work Is», The Journal of Philosophy 77/1: 5-28.

Limido-Heulot, Patricia. 2001. «Phénoménologie et ontologie chez Roman Ingarden», introduction à Roman Ingarden, Husserl, la controverse Idéalisme - Réalisme, 12. Paris: Vrin.

Mazzoni, Augusto. Avril 1998. «Perspectives of music theory in Waldemar Conrad's aesthetics", Axiomathes 9/1: 223-233.

Nef, Frédéric. 2006. «Ontologie de l'objet, théorie des propriétés et théorie des ensembles: quelques problèmes et perspectives", Revue internationale de philosophie 2/236: 181 .

Peterson, Richard A. et Roger M. Kern. 1996. "Changing Highbrow Taste: From Snob to Omnivore», American Sociological Review 61/5: 900-907.

Pouivet, Roger. 2010. Philosophie du rock. Une ontologie des artefacts et des enregistrements. Paris: PUF. 
Rieser, Max . Été 1971. "Roman Ingarden and His Time», The Journal of Aesthetics and Art Criticism 29/4: 443-452.

Schaeffer, Jean-Marie. 1999. Pourquoi la fiction? Paris: Seuil, Smith, Barry et David M. Mark.( Sans date). «Do Mountains Exist? Ontology of Landforms and Topography ", 14. http://ontology.buffalo.edu/smith/ articles (consulté le 1o novembre 2010).

Stévance, Sophie. 2009. Duchamp, compositeur. Paris: L'Harmattan.

Sullivan, Oriel et Tally Katz-Gerro. 2007. «The Omnivore Thesis Revisited: Voracious Cultural Consumers", European Sociological Review 23/2: 123-137.

Tagg, Philip. 2000. "The Work": An Evaluative Charge». The Musical Work: Reality or Invention, sous la dir. de M. Talbot, 153-167. Trowbridge: Liverpool University Press.

Vázquez, Osvaldo. "El espacio en música», http://osvazqz.wordpress.com (consulté le 10 novembre 2010).

Wollheim, Richard. 1980. Art and its Objects. Cambridge: Cambridge University Press.

Wolterstorff, Nicholas. Mai 1975. «Toward an Ontology of Art Works», Nô̂s 9/2:115-142.

Web: http://www.radioclassique.fr/ (consulté le 8 novembre 2010).

\title{
RÉSUMÉ
}

Qu'est-ce qu'une ceuvre musicale? La question ontologique soulevée par Roman Ingarden dans sa critique de la phénoménologie de Husserl a de nombreux points communs avec la querelle du "platonisme musical" déployée dans les années quatre-vingt par des philosophes analytiques tels que Jerrold Levinson et Peter Kivy. En 1992, Lydia Goehr a proposé une historicisation radicale du concept d'œuvre musicale, sans pour autant liquider un projet ontologique qui, tout récemment, insiste dans les réflexions de Roger Pouivet à propos de "l'œuvre musicale rock". Cet article fait le point sur ces débats philosophiques avant de signaler la crise sociologique de la notion d'œuvre liée au virage numérique et à la fragmentation du répertoire classique selon les standards de l'industrie culturelle. Reprenant la définition d'Ingarden de "l'objet purement intentionnel" à partir de cet ébranlement du sens commun, il compare pour finir le statut ontologique de l'œuvre musicale à celui des êtres de fiction, un personnage de roman par exemple, ainsi qu'à celui d'entités qui, telles les montagnes évoquées par la "théorie granulaire de la réalité" de Barry Smith, résultent de la projection d'une pratique langagière sur la surface sonore du monde.

\begin{abstract}
What is a musical work? This ontological question raised by Roman Ingarden in his work critical of Husserl's phenomenology presents numerous common aspects with the issue of "musical Platonism" which was developed in the 1980 os by analytic philosophers such as Jerrold Levinson and Peter Kivy. In 1992, Lydia Goehr put forward a radical historicization of the concept of musical work, without nonetheless discarding an ontological project, recently stressed in Roger Pouivet's considerations on the "rock
\end{abstract}


musical work." This essay sums up these philosophical debates and underlines the sociological crisis of the concept of work in the context of the digital revolution and the fragmentation of the classical repertoire required by the cultural industry. Considering Ingarden's definition of the "purely intentional object" from the point of view of the common sense's destabilization, this study compares the ontological status of the musical work to that of fictional beings, such as a novel character, as well as to that of entities, such as the mountains echoed by Barry White's "granular theory of reality," emerging from the projection of language usages onto the audible world. 